\title{
Tissue-based coronary surgery simulation: Medical student deliberate practice can achieve equivalency to senior surgery residents
}

\author{
Jonathan C. Nesbitt, MD, Jamii St Julien, MD, Tarek S. Absi, MD, Rashid M. Ahmad, MD, \\ Eric L. Grogan, MD, MPH, Jorge M. Balaguer, MD, Eric S. Lambright, MD, Stephen A. Deppen, MA, MS, \\ Huiyun $\mathrm{Wu}, \mathrm{PhD}$, and Joe B. Putnam, MD
}

\begin{abstract}
Objective: The study objective was to assess the impact of dedicated instruction and deliberate practice on fourth-year medical students' proficiency in performing a coronary anastomosis using a porcine heart model, compared with nonsimulator-trained senior general surgery residents.

Methods: Ten fourth-year medical students were trained to perform a coronary anastomosis using the porcine simulator. Students trained for 4 months using deliberate practice methodology and one-on-one instruction. At the end of the training, each student was filmed performing a complete anastomosis. Eleven senior general surgery residents were filmed performing an anastomosis after a single tutorial. All films were graded by 3 independent cardiac surgeons in a blinded fashion. The primary outcome was the median final score (range, 1-10) of a modified Objective Structured Assessment of Technical Skill scale. The secondary outcome was time to completion in seconds. Statistical analysis used both parametric (Student $t$ test) and nonparametric (Wilcoxon rank-sum) methods.
\end{abstract}

\begin{abstract}
Results: The median combined final score for medical students was 3 (interquartile range, 2.3-4.8), compared with 4 (interquartile range, 3.3-5.3) for residents $(P=.102)$. The overall median individual final scores were 3 (interquartile range, 2-6) for grader 1, 3 (interquartile range, 2-5) for grader 2, and 4 (interquartile range, 3-5) for grader 3. For each individual grader, there was no difference in median final scores between medical students and residents. The mean time to completion was 792.7 seconds (95\% confidence interval, 623.4-962) for medical students and 659 seconds (95\% confidence interval, 599.1-719) for residents $(P=.118)$.

Conclusions: Dedicated instruction of fourth-year medical students with deliberate and distributed practice of microvascular techniques using a porcine end-to-side coronary artery anastomosis simulation model results in performance comparable to that of senior general surgery residents. These results suggest that focused tissue simulator training can compress the learning curve to acquire technical proficiency in comparison with realtime training. (J Thorac Cardiovasc Surg 2013;145:1453-9)
\end{abstract}

Simulation training in surgery continues to evolve as an important component in the resident educational experience. The benefits and challenges, both real and theoretic, are well documented in multiple specialties, including cardiothoracic surgery (CTS). ${ }^{1-6}$ Training programs in CTS have increasingly used simulation as part of the core curriculum to heighten key technical skills that can transport to the operating room. Highfidelity and tissue simulators have been found to help

\footnotetext{
From the Vanderbilt University Medical Center, Department of Thoracic Surgery, Nashville, Tenn.

Disclosures: Authors have nothing to disclose with regard to commercial support.

Read at the 38th Annual Meeting of The Western Thoracic Surgical Association, Maui, Hawaii, June 27-30, 2012.

Received for publication June 19, 2012; revisions received Feb 5, 2013; accepted for publication Feb 14, 2013; available ahead of print March 18, 2013.

Address for reprints: Jonathan C. Nesbitt, MD, 1313 21st Ave S, 609 Oxford House,

Nashville, TN 37232 (E-mail: jon.nesbitt@ vanderbilt.edu).

$0022-5223 / \$ 36.00$

Copyright (c) 2013 by The American Association for Thoracic Surgery

http://dx.doi.org/10.1016/j.jtcvs.2013.02.048
}

provide an optimal learning platform that enhances the educational process. ${ }^{3,7,8}$

In 2007, the Joint Council for Thoracic Surgery Education convened to review trends and concerns in thoracic surgical education. From this meeting, the Council provided thoughtful recommendations to address resident work hour restrictions, the decline in applicants to traditional programs, and resident performance on board examinations. ${ }^{9,10}$ Foremost in the recommendations was the need to reform CTS education with simulation as a primary focus. ${ }^{10-12}$

Concurrent to the Joint Council for Thoracic Surgery Education recommendations, the American Board of Thoracic Surgery approved the development and implementation of a 6-year integrated cardiothoracic curriculum (I-6) for CTS. $^{13,14}$ To date, 18 programs have initiated I-6 residencies, although the effectiveness of the programs remains unproven. Questions remain regarding the program's ability to condense the educational, clinical, and operative experience necessary for the safe and independent 


\section{Abbreviations and Acronyms \\ CTS = cardiothoracic surgery \\ IQR = interquartile range \\ OSATS $=$ Objective Structured Assessment of Technical Skill}

practice of the specialty at the completion of training. An additional concern is the need or ability to train medical students with minimal surgical experience in the skills of microvascular techniques. Simulation training has been proposed as one potential solution to these concerns.

We evaluated the acquisition of microvascular suturing skills of fourth-year medical students using short-duration simulation training. To provide a degree of validation, the study group was compared with senior-level general surgery residents with microvascular surgery skills acquired in a real-time setting during their years of residency education.

\section{MATERIALS AND METHODS Study Design}

This study was a single-center, blinded, prospective, randomized intervention trial. The study was approved by the Vanderbilt University Medical Center Internal Review Board. Inclusion criteria for the study participants were as follows: (1) fourth-year medical students with little or no training in suturing techniques and no training in microvascular suturing techniques, and (2) senior general surgery residents with more than 3 years of general surgery experience including 6-week rotations on the transplant, vascular, and pediatric surgery services where microvascular surgery experiences were obtained. (A sampling of the Vanderbilt University Medical Center senior per-resident caseload experience for full credit during this time showed the following: $>10$ major vascular cases, $>25$ vascular access cases, $>40$ microvascular anastomoses while performing renal transplantation, and $>30$ pediatric cases requiring loupe dissection.) None of the residents had practiced microvascular techniques in a simulated environment.

This study was voluntary and discussed with potential candidates before entry. Each student had expressed an interest in a career in surgery or simply wanted to learn suturing skills. Consent was obtained after explanation of the study by the primary investigator. Ten students and 11 residents consented to the study. All students had minimal to no prior experience in suturing techniques.

The study period extended weekly or biweekly over 4 months. During the initial 2 instruction sessions, students were taught the fundamentals of suturing techniques, and they practiced sewing on cloth material. They were given a needle holder, forceps, scissors, and suture material to practice at home. For the third session, the students practiced closing aortotomies on segments of fresh porcine aortas using 3-0 polypropylene suture. During the remaining sessions, students performed coronary artery bypass grafting on fresh, ex vivo porcine hearts. Students were given intentional, one-on-one detailed instruction of all components of microvascular technique during every anastomosis and were provided practice time with coaching of specific aspects: hand mechanics, hand/wrist/finger position and movement with each instrument (forceps and needle holder), tissue handling, needle angles, economy of motion techniques, needle-in-tissue movements, body position, needle/suture placement and spacing, suture tension, traction-countertraction maneuvers, and communication with the assistant. Instructive feedback was provided during each session. At each 2-hour coronary artery teaching session, 4 to 6 students were present. Each student attended from 5 to as many as 7 sessions, therefore spending
10 to 14 hours total in the coronary suturing laboratory. During each session, the students alternatively performed the anastomosis or first assisted during the procedures. Assisting skills were taught in this manner. All students ultimately performed at least 12 anastomoses (range, 12-18). No practicing was permitted between the coronary suturing sessions to ensure the quality of the training and to reduce the variability of the practice time. At the completion of training, the final anastomosis was filmed. The residents received no instruction and had no prior experience with the porcine model. With the same instructor who taught the students, each resident was allowed to practice 1 anastomosis before being filmed.

During training and filming of all participants, all coronary anastomoses were performed in an identical fashion. Optical magnification was not permitted. The canine saphenous vein was sewn to a $10-\mathrm{mm}$ anterior descending coronary arteriotomy. All anastomoses were accomplished using 7-0 polypropylene suture. The first 3 bites were taken in an open fashion at the anastomotic heel, placing all arterial sutures "inside out" and all vein sutures "outside in." Suturing continued in a counterclockwise sequence until completion. During filming, the same first assistant was used for all participants. Only the hands and operative field were filmed. The time to completion was recorded from the beginning of the first suture placement to completion of the last suture placement. Tying was not included in time measurement because the objective of the study was to measure only suturing proficiency. The filmed sessions were coded and electronically secured in the Department of Thoracic Surgery and then reviewed by 3 cardiac surgeons who were blinded to the randomization of the participants and who independently graded the performance of the participants using modified Objective Structured Assessment of Technical Skill (OSATS) grade sheets (Figure 1). ${ }^{15,16}$

\section{Statistical Analysis}

The primary outcome was the final score of the modified OSATS scale (Figure 1). This score ranged from 1 to 10 , was selected by each grader on the grading sheet itself, and was treated as a discrete variable in our analyses. We combined the scores from the 3 independent individual graders to obtain an average to the final. Wilcoxon rank-sum test was used to compare the distribution of scores between the medical student and the resident study group. We also took the intergrader variability into consideration in evaluating the 2 methods. We first performed a kappa analysis to see the agreement among the graders and then performed a sensitivity analysis, in which the data by each individual grader were analyzed and the results were compared with those from combined data to examine whether the 2 approaches would change the results substantially. The secondary outcome was time to completion (in seconds) and was compared between study groups using the Student $t$ test.

We also compared the scores of each individual component of the modified OSATS scale (range, 1-5) independently for each grader and as a combined (averaged) score for each component between study groups using the Wilcoxon rank-sum test. A Bonferroni correction was performed because of the multiple comparisons ( 8 comparisons per grader) (Table 1).

All tests were 2-tailed, and all computations were done using Stata Statistical Software: Release 11 (StataCorp LP, College Station, Tex).

\section{RESULTS}

The median combined final score was 4 (interquartile range [IQR], 2.7-4.3), and mean time to completion was 719 seconds (12 minutes). The combined median final score was 3 (IQR, 2.3-4.8) for the students and 4 (IQR, 3.3-5.3) for the residents $(P=.102)$ (Table 2; Figure 2). The mean time to completion was 792.7 seconds $(95 \%$ confidence interval, 623.4-962) for the students and 659 seconds (95\% confidence interval, 599.1-719) for the residents $(P=.118)$ (Figure 3). 
ID Number :

Cardiothoracic Simulation Grade Sheets

Coronary artery anastomosis

Time to completion: seconds (from time of arteriotomy to anastomosis completion).

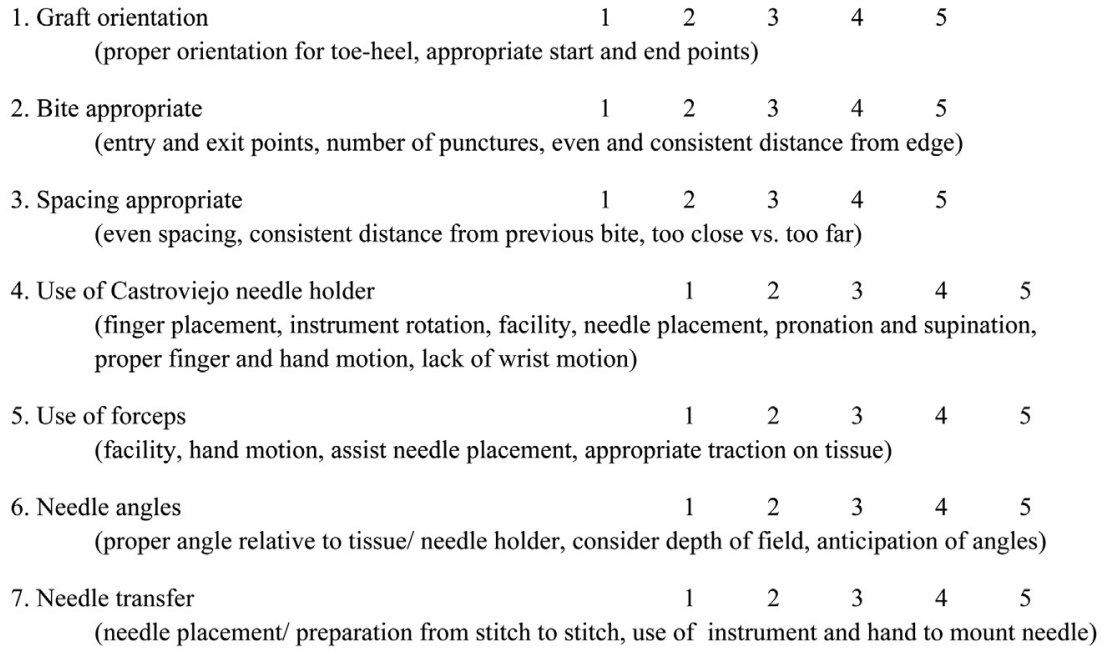

FIGURE 1. Grading sheets. Modified from Fann and colleagues. ${ }^{16}$

Assessment of the intergrader reliability among the 3 graders for the median final score revealed a kappa statistic of -0.04 , implying poor agreement. However, the sensitivity analysis found that the 3 individual graders had the same pattern of score distribution across the 2 groups and came to the same conclusion as the combined score. For the results to be more informative, we reported both average score and individual scores to demonstrate the performance of the 2 groups of examiners (Table 2 and Figure 4). Table 1 shows the results of each individual component of the modified OSATS scale.

\section{DISCUSSION}

The coronary anastomosis remains the fundamental procedure for coronary revascularization. In contemporary training venues, residents acquire skills through real-time experience. Proof of proficiency is not frequently required before the action. We believe that simulation training 
TABLE 1. Individual grading components

\begin{tabular}{|c|c|c|c|}
\hline Grading category & $\begin{array}{c}\text { Medical students, } \\
\text { median (IQR) }\end{array}$ & $\begin{array}{c}\text { Senior residents, } \\
\text { median (IQR) }\end{array}$ & $P$ value* \\
\hline \multicolumn{4}{|l|}{ Grader 1} \\
\hline Graft orientation & $2(2-3)$ & $3(2-3)$ & 1.00 \\
\hline Bite & $1(1-2)$ & $2(2-3)$ & .112 \\
\hline Spacing & $1(1-2)$ & $2(2-3)$ & 1.00 \\
\hline Use of Castro & $2(2-2)$ & $2(2-3)$ & 1.00 \\
\hline Use of forceps & $2(1-2)$ & $2(2-3)$ & 1.00 \\
\hline Needle angles & $1.5(1-2)$ & $2(1-3)$ & 1.00 \\
\hline Needle transfer & $2(1-2)$ & $2(2-3)$ & 1.00 \\
\hline Economy of time & $2(1-2)$ & $2(1-4)$ & 1.00 \\
\hline \multicolumn{4}{|l|}{ Grader 2} \\
\hline Graft orientation & $3(3-3)$ & $3(3-3)$ & 1.00 \\
\hline Bite & $2.5(2-3)$ & $3(2-3)$ & 1.00 \\
\hline Spacing & $2.5(2-3)$ & $3(3-3)$ & 1.00 \\
\hline Use of Castro & $2(2-2)$ & $3(2-3)$ & .584 \\
\hline Use of forceps & $2(2-3)$ & $2(2-3)$ & 1.00 \\
\hline Needle angles & $2.5(2-3)$ & $3(2-3)$ & 1.00 \\
\hline Needle transfer & $2(2-3)$ & $2(2-3)$ & 1.00 \\
\hline Economy of time & $2(2-3)$ & $3(2-3)$ & 1.00 \\
\hline \multicolumn{4}{|l|}{ Grader 3} \\
\hline Graft orientation & $3(3-3)$ & $3(3-3)$ & 1.00 \\
\hline Bite & $2(2-3)$ & $3(2-3)$ & .888 \\
\hline Spacing & $3(2-3)$ & $3(3-3)$ & .808 \\
\hline Use of Castro & $3(2-3)$ & $3(3-3)$ & 1.00 \\
\hline Use of forceps & $3(3-3)$ & $3(3-3)$ & 1.00 \\
\hline Needle angles & $3(3-3)$ & $3(3-3)$ & 1.00 \\
\hline Needle transfer & $2(2-3)$ & $3(3-3)$ & .04 \\
\hline Economy of time & $2(2-3)$ & $3(2-3)$ & .376 \\
\hline \multicolumn{4}{|l|}{ Combined score } \\
\hline Graft orientation & $2.7(2.7-3)$ & $2.7(2.7-3)$ & 1.00 \\
\hline Bite & $2(1.7-2.3)$ & $2.7(2-3)$ & .464 \\
\hline Spacing & $2.3(2-3)$ & $3(2.7-3)$ & 1.0 \\
\hline Use of Castro & $2(2-3)$ & $2.7(2.3-3)$ & .872 \\
\hline Use of forceps & $2.5(2-3)$ & $2.7(2.3-3)$ & 1.00 \\
\hline Needle angles & $2.5(2-2.7)$ & $2.7(2.3-3)$ & 1.00 \\
\hline Needle transfer & $2(1.7-2.3)$ & $2.7(2.3-3)$ & .144 \\
\hline Economy of time & $2(1.7-2.7)$ & $2.7(2.3-3)$ & .68 \\
\hline
\end{tabular}

$I Q R$, Interquartile range. *Bonferroni-adjusted $P$ values are presented.

provides a means to develop proficiency before clinical application.

In this small single institution study, medical students who received specific short-term technical instruction with deliberate practice and formative feedback performed at a level comparable to that of senior-level general surgery residents when accomplishing a coronary anastomosis on a porcine model. Time to completion of the anastomosis and the quality of the anastomosis were comparable. The residents had a nonstatistically significant trend to shorter anastomosis time and a smaller standard deviation (on average) than medical students. Other experiential learning by the residents likely attributed to this finding.

The porcine cardiac anastomosis model provided a controlled, low-stress environment for students to learn and
TABLE 2. Final score measures compared between study groups

\begin{tabular}{|c|c|c|c|c|}
\hline Outcome measure & $\begin{array}{c}\text { All } \\
\text { participants } \\
(\mathrm{N}=\mathbf{2 1})\end{array}$ & $\begin{array}{l}\text { Medical } \\
\text { students } \\
(\mathrm{N}=10)\end{array}$ & $\begin{array}{c}\text { Senior } \\
\text { residents } \\
(\mathrm{N}=11)\end{array}$ & $P$ value \\
\hline $\begin{array}{l}\text { Grader } 1 \text { final score, } \\
\text { median (IQR) }\end{array}$ & $3(2-6)$ & $2(1-4)$ & $4(2-7)$ & .211 \\
\hline $\begin{array}{l}\text { Grader } 2 \text { final score, } \\
\text { median (IQR) }\end{array}$ & $3(2-5)$ & $2.5(2-5)$ & $3(3-5)$ & .228 \\
\hline $\begin{array}{c}\text { Grader } 3 \text { final score, } \\
\text { median (IQR) }\end{array}$ & $4(3-5)$ & $3.5(3-4)$ & $4(3-5)$ & .324 \\
\hline $\begin{array}{l}\text { Combined final score, } \\
\text { median (IQR) }\end{array}$ & $4(2.7-4.3)$ & $3(2.3-4.8)$ & $4(3.3-5.3)$ & .102 \\
\hline
\end{tabular}

practice microvascular techniques. The porcine model also allowed for the use of real tissue for optimal handling of the target sites that is not provided with artificial structures. All participants thought the tissue simulator provided a realistic model on which to train.

This study was designed to run for only 4 months, and a set number of practice anastomoses was not required. We chose this structure because (1) the student curriculum constraints allowed only a 4-month window to complete the study; (2) time in practice, rather than events (anastomoses), is the current standard of training; (3) numbers of anastomoses performed or time to practice does not necessarily equate to proficiency; and (4) short-term, focused training improves the operator's ability to perform coronary anastomoses with no set criteria of numbers performed. ${ }^{3,16}$

Data were not collected at intervals during training to document the progress of suturing proficiency. Only the final anastomosis was filmed and graded. Because the objective of the study was to compare with residents the level of suturing skill acquired through short-term training, the rate of skill acquisition of the students was not specifically measured. Video recordings were used to document and provide anonymity to the participant's final anastomosis. The video assessment was performed in a standard manner for all participants. Beard and colleagues ${ }^{17}$ reported the feasibility and reliability of video recording in the assessment of surgical competence, particularly before undertaking an actual operation. They also noted that deconstruction of operations into their component parts enable trainees to practice on simple simulations that represent component parts of the procedure.

Senior-level general surgery residents were chosen for the study because they (1) provided a midlevel benchmark for comparison, (2) had microvascular surgery experience that was obtained real-time as part of their residency education, and (3) had no simulation training in microvascular techniques. Although surgical experiences varied between residents, there was the expectation that microvascular techniques had been performed 


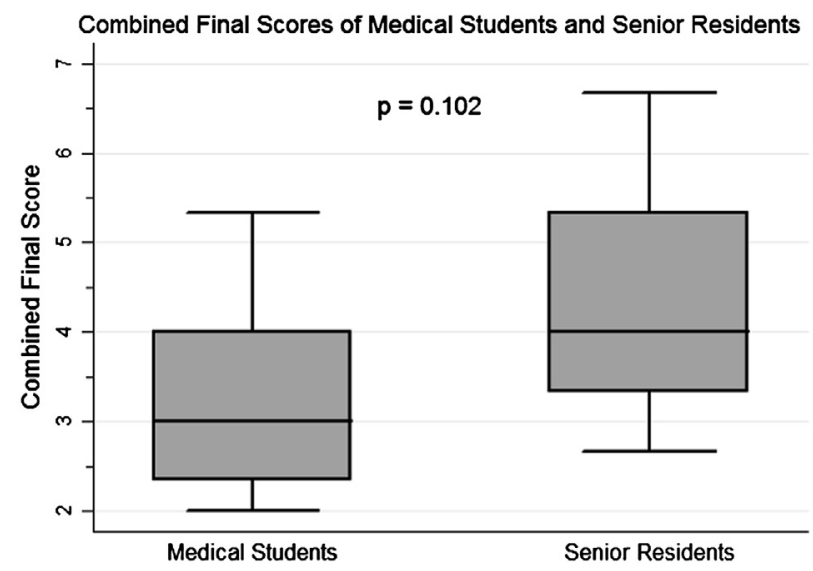

FIGURE 2. Comparison of combined final scores between study groups.

during actual operations and the acquired skills were commensurate to those of a senior resident level after 3 years of training.

\section{Study Limitations}

One limitation with the study is the small sample size. Although a nonsignificant statistical value was achieved, a larger cohort would have allowed for detection of more subtle differences between groups. It will be important for future studies to incorporate larger study groups, so that our findings can be supported with greater statistical power.

As an additional post hoc reliability check of our results, we conducted a permutation test on the combined final score and maximum time to complete the exercise. ${ }^{18}$ The permutation test is a relatively simple method to estimate the accuracy of our significance estimate in small sample sets. We repeated the permutation test 5000 times and estimated the distribution of the Wilcox sum-rank test $P$ values for the combined final score outcome from those repetitions. The permutation-adjusted $P$ value was .105 , suggesting that

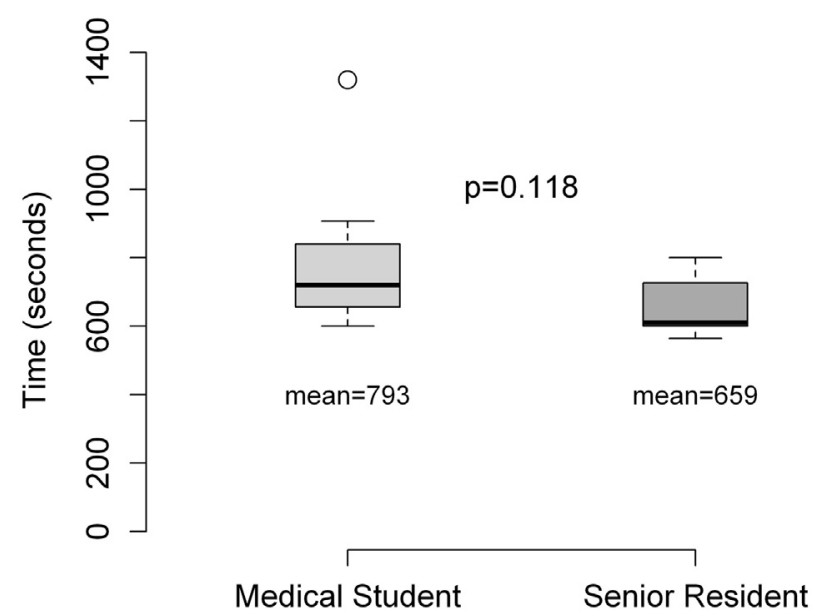

FIGURE 3. Comparison of mean time to completion of anastomosis between study groups.

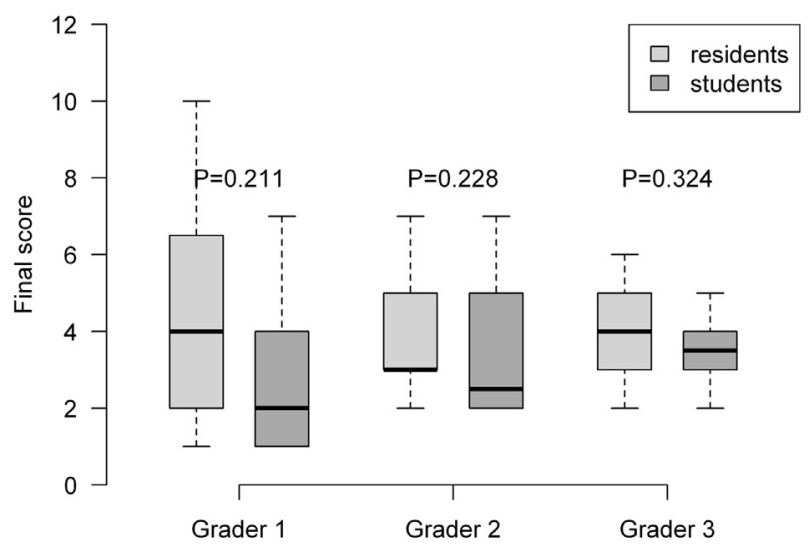

FIGURE 4. Grader scores by training group. The plot shows the difference in the final score between students and residents evaluated by each one of the 3 graders. A $P$ value was computed for the comparison of students versus residents for each particular grader.

our observed $P$ value of .102 was well within the expected distribution of $P$ values. Likewise, we conducted the permutation test on the maximum time to completion of the exercise. The permutation-adjusted $P$ value was .079 , which was also consistent with the $P$ value of .086 observed in the experimental data. In short, the additional permutation test examining the distribution of $P$ values further supports our findings despite the small sample size of our study.

The results might have reflected more familiarity of the students to the porcine model because after 12 to 18 anastomoses, performances of the students were comparable to those of the residents. Training to the trainer is always a consideration when selecting a simulation model and choosing the venue in which to train. Despite every effort to create a trainer and training environment to which the residents could quickly adapt, the students certainly had more hours invested on the trainer. However, the porcine tissue trainer was chosen for several reasons: (1) Real tissue was more familiar to the residents than synthetic material; (2) a microvascular anastomotic setup with which the residents were familiar was used; (3) large porcine hearts were used because of the large anterior descending coronary arteries with anastomotic sizes comparable to those performed during resident training experiences; and (4) instruments used for suturing were identical to those used by the residents in the operating room. Therefore, every effort was made to reduce the possibility of trainer bias by providing as much uniformity and familiarity as possible for each group. Although it is hoped and expected that residents in training will improve their technical skills during each year of training and with each case, rarely if ever do they experience focused, one-on-one skill training in a simulation environment. The method of focused, distributed, and deliberate practice with immediate formative feedback occurred for the students in this study. Although training to the trainer may have played a role in the results, it is believed the methodology of the simulation 
training made the difference and showed that the time for skill acquisition can be compressed.

The rating scale used in this study may not have been as complete as the scales used in other studies. The scoring tool was a modified version of the OSATS that included a global rating scale and a task checklist. Specific task components within the tool were the same as those used in grading tools during the Thoracic Surgery Directors Association Boot Camps. ${ }^{3,16}$ The global rating scale was expanded to allow the graders a broader range of scoring based on their own experiences. Offering a wider range for global assessment was believed to be important because a more inexperienced group of trainees was being graded. For this study, a pass/ fail grade was not included in the assessment because it would not be applicable to the limited level of training.

There was lack of standardized training for graders to reduce intergrader variability. Faculty assessors were cardiac surgeons with $100 \%$ cardiac surgery practice and who had active participation in our residency education program. They evaluated participants at similar assessment levels, and trends toward improved performance for surgical residents were identified. Faculty assessors were not given specific training in assessment methods; however, they were allowed to judge the level of proficiency on the basis of a Dreyfus model for skill acquisition used for routine evaluation of our CTS residents. ${ }^{19}$ This resulted in significant grader variability as shown in Figure 3. On initial assessment, the poor interrater agreement seems to decrease the validity of our primary conclusion; however, a sensitivity analysis evaluation of the results of each grader separately (Table 2 and Figure 4) revealed that they all came to the same conclusion. Each individual grader was consistent in grading each participant and did not differentiate the quality of surgical technique between the 2 study groups. Thus, the validity of our results is supported. For future studies, a more regimented protocol of training for the faculty assessors before video grading should improve interrater agreement and minimize any concerns for study validity.

\section{CONCLUSIONS}

In our 4-month simulation program, fourth-year medical students learned microvascular suturing skills. With direct instruction, immediate and formative feedback, and distributed and deliberate practice using a porcine coronary anastomosis model, students performed at a skill level comparable to that of senior level general surgery residents.

\section{References}

1. Cook DA, Hatala R, Brydges R, Zendejas B, Szostek JH, Wang AT, et al. Technology-enhanced simulation for health professions education: a systematic review and meta-analysis. JAMA. 2011;306:978-88.

2. Sturm LP, Windsor JA, Cosman PH, Cregan P, Hewett PJ, Maddern GJ. A systematic review of skills transfer after surgical simulation training. Ann Surg. 2008;248:166-79.
3. Fann JI, Calhoon JH, Carpenter AJ, Merrill WH, Brown JW, Poston RS, et al. Simulation in coronary artery anastomosis early in cardiothoracic surgical residency training: the Boot Camp experience. J Thorac Cardiovasc Surg. 2010;139:1275-81.

4. Desender LM, Van Herzeele I, Aggarwal R, Vermassen FE, Cheshire NJ. Training with simulation versus operative room attendance. J Cardiovasc Surg (Torino). 2011;52:17-37.

5. Aggarwal R, Mytton OT, Derbrew M, et al. Training and simulation for patient safety. Qual Saf Health Care. 2010;19(Suppl 2):i34-43.

6. Reznick RK. Surgical simulation: a vital part of our future. Ann Surg. 2005;242: 640-1.

7. Joyce DL, Dhillon TS, Caffarelli AD, Joyce DD, Tsirigotis DN, Burdon TA, et al. Simulation and skills training in mitral valve surgery. J Thorac Cardiovasc Surg. 2011;141:107-12.

8. Ramphal PS, Coore DN, Craven MP, Forbes NF, Newman SM, Coye AA, et al. A high fidelity tissue-based cardiac surgical simulator. Eur J Cardiothorac Surg. 2005;27:910-6.

9. Prasad SM, Massad MG, Chedrawy EG, Snow NJ, Yeh JT, Lele H, et al. Weathering the storm: how can thoracic surgery training programs meet the new challenges in the era of less-invasive technologies? J Thorac Cardiovasc Surg. 2009;137:1317-26.

10. Chitwood WR Jr, Spray TL, Feins RH, Mack MJ. Mission critical: thoracic surgery education reform. J Thorac Cardiovasc Surg. 2008;136:812-3.

11. Feins RH. Expert commentary: cardiothoracic surgical simulation. J Thorac Cardiovasc Surg. 2008;135:485-6.

12. Carpenter AJ, Yang SC, Uhlig PN, Colson YL. Envisioning simulation in the future of thoracic surgical education. J Thorac Cardiovasc Surg. 2008;135:477-84.

13. American Board of Thoracic Surgery. Booklet of information. Available at: http:// www.abts.org/documents/PDF/2010_Booklet_of_Information.pdf. Accessed 2012.

14. Kron IL. Changes in thoracic surgery training. Am Surg. 2007;73:155-6.

15. Reznick R, Regehr G, MacRae H, Martin J, McCulloch W. Testing technical skill via an innovative "bench station" examination. Am J Surg. 1997;173:226-30.

16. Fann JI, Caffarelli AD, Georgette G, Howard SK, Gaba DM, Youngblood P, et al. Improvement in coronary anastomosis with cardiac surgery simulation. J Thorac Cardiovasc Surg. 2008;136:1486-91.

17. Beard JD, Jolly BC, Newble DI, Thomas WE, Donnelly J, Southgate LJ. Assessing the technical skills of surgical trainees. Br J Surg. 2005;92:778-82.

18. Good P. Permutation Tests: A Practical Guide to Resampling Methods for Testing Hypothesis. New York: Springer; 1994.

19. Dreyfus SE. The five-stage model of adult skill acquisition. Bull Sci Technol Soc. 2004;24:177-81.

\section{Discussion}

Dr James Fann (Palo Alto, Calif). Dr St Julien, I congratulate you on a very informative and much-needed study in the field of simulation and deliberate practice. You and your colleagues are to be commended for this effort.

In evaluating medical students and resident training in cardiothoracic surgery, we need to evaluate not only the cognitive skills that we do very well but also the psychomotor skills. We have always been very good at addressing the cognitive ability through the in-training examination and, subsequently, through the efforts of the American Board of Thoracic Surgery. More recently, the Thoracic Surgery Directors Association and the Joint Council on Thoracic Surgery Education have focused on technical skills, which, in part, is impetus for the study.

I have 3 questions and I will pause for your response after each. You noted that the students performed a minimum of 12 vascular anastomoses, but no practice was allowed outside the laboratory or between sessions. Acknowledging the importance of formative feedback, which is well documented in the study, would you consider the lack of practice outside of the laboratory to be consistent with the concept of deliberate practice? And is it possible that, had the students been given additional practice time, they might have demonstrated equivalent and perhaps superior skills to the general surgery residents? 
Dr St Julien. Thank you, Dr Fann. That is a very good question. You are $100 \%$ correct. The term deliberate practice is different than what we did here. The students did not have the option of practicing on their own for a couple of reasons: One, because we could not force them to-it was a purely elective study-and if we would have allowed them to, it would have been a huge variation from student to student as far as how much practice they got; and two, we wanted to really be able to measure the amount of practice done, so restricting it to the practice sessions where we could actually count the anastomoses was important for us. That being said, the practice that they did get, though it was instructed in the session, was very deliberate and was very detailed. While, yes, I do think that the students probably would have performed better if they had more time to practice even on their own, they got quite a substantial amount of practice even in 1 anastomosis session.

Dr Fann. What has always been a concern in simulation-based learning is the model that is used for practice and assessment. For instance, in contrast to the students in the study, the general surgery residents were allowed to have 1 session to become familiar with the model, and shortly thereafter they did 1 session that was video recorded and assessed. The main criticism of such an approach is how do you know you are not training to the simulator; that is, with greater experience using a particular simulator, one would expect to do much better. Are we handicapping the general surgery residents by limiting their exposure to the simulator?

Dr St Julien. Another great comment. This was a high-fidelity real-tissue simulator model. We felt that we did not really have to deal with that issue because the residents all had experience dealing with tissue. They were trained on real tissue. They were very familiar with the forceps and all the materials used, so what they had to learn in that 1 session was just the rules of the anastomosis for our particular study and the flow with which we wanted it to be done. It was very different than if they were to start training on a synthetic model where they would have to actually learn the simulator in which the medical students who had all the training on that synthetic model would have an advantage.

Dr Fann. Finally, you stated that not all students were able to attend all the sessions and that some students practiced more than others. You also noted that more practice and more anastomoses did not always result in better performance. My question is given your observation and the understanding of the confounders that go into assessment and skills training, when you describe the concept of "innate ability" did you notice any characteristics that were predictive of performance?

Dr St Julien. Of course each student and each resident will bring his or her own innate ability and we actually did notice that. Some students picked it up very quickly and some took longer. The first 5 or 6 anastomoses showed the fastest learning curve in general and the improvement from each anastomosis thereafter sort of plateaued a bit. You are absolutely right: Each student was pretty variable in how quickly he or she picked things up. It is just one of those issues that a study like this has to acknowledge and accept.

Dr Fann. I think there is a fair amount of room for future research in this area, especially in evaluating the motivational components in surgical training and what abilities the resident brings to the training process. I appreciate the Association for the privilege of discussing this paper. 